TRANSACTIONS OF THE

AMERICAN MATHEMATICAL SOCIETY

Volume 351, Number 3, March 1999, Pages 1075-1094

S 0002-9947(99)02082-6

\title{
ON THE NON-VANISHING OF CUBIC TWISTS OF AUTOMORPHIC L-SERIES
}

\author{
XIAOTIE SHE
}

\begin{abstract}
Let $f$ be a normalised new form of weight 2 for $\Gamma_{0}(N)$ over $\mathbb{Q}$ and $F$, its base change lift to $\mathbb{Q}(\sqrt{-3})$. A sufficient condition is given for the nonvanishing at the center of the critical strip of infinitely many cubic twists of the $L$-function of $F$. There is an algorithm to check the condition for any given form. The new form of level 11 is used to illustrate our method.
\end{abstract}

\section{INTRODUCTION}

There has been a tremendous amount of recent research on the nonvanishing of $L$-functions. On $G L(2)$, the first results are due to Shimura [15], who proved that a given $L$-function can be twisted by a character of finite order so that the twisted $L$-function does not vanish at a certain point. Shimura's nonvanishing results were generalized by Rohrlich [14]. The study of the nonvanishing of quadratic twists rather than arbitrary finite order twists was started by Goldfeld, Hoffstein and Patterson [7] for the CM case, and in greater generality by Waldspurger [16]. This was later complemented by the work of Bump, Friedberg and Hoffstein [2], [3], [5] using metaplectic Eisenstein series and the Rankin-Selberg method. Alternately, analytic number theoretic methods have been used by Murty and Murty [12] and by Iwaniec [10] to obtain nonvanishing results. The first results on the non-vanishing of cubic twists were obtained by Lieman [11]. He applied the theory of automorphic forms on the cubic cover of $G L(3)$ to the $L$-series of the CM elliptic curve $x^{3}+y^{3}=1$. In this way he obtained a non-vanishing result for cubic twists of the $L$-series of the automorphic form corresponding to the curve. However, because the curve has complex multiplication, the $L$-series in this instance is a $G L(1)$ Hecke $L$-series with grossencharacter. It is this particular fact which made the $G L(3)$ theory applicable.

In this paper we will give the first non-vanishing results for cubic twists of autmorphic $L$-series on $G L(2)$ that are not lifts from $G L(1)$. In particular, we will show that if $f$ is the new form of weight 2 and level 11, then infinitely many cubic twists of the $L$-series of $f$ are non-zero at the center of the critical strip. It then follows as an immediate corollary that there are infinitely many cubic extensions $K$ of $\mathbb{Q}(\sqrt{-3})$ such that the analytic rank of $E=X_{0}(11)$ over $K$ is zero.

Our approach gives a method of obtaining a similar result for any given $G L(2)$ automorphic form. However, for reasons that will be described below, there is one step in the computation which can be verified for any given form, but which cannot yet be done in general.

Received by the editors September 27, 1996 and, in revised form, February 14, 1997.

1991 Mathematics Subject Classification. Primary 11F66; Secondary 11F70, 11M41, 11N75.

(C)1999 American Mathematical Society 
Our work is based on a technique recently introduced in [5]. This method involves the convolution of an automorphic form $f$ with an Eisenstein series on the double cover of $G L(2)$. We have applied this technique to a similar Eisenstein series on the cubic cover of $G L(2)$ and have succeeded in obtaining information about the non-vanishing of cubic twists of the $L$-series of $f$.

However, in order for the cubic Eisenstein series to be defined, the ground field must include the cube roots of unity. Thus we have constructed $F$, the base change of $f$ to $\mathbb{Q}(\sqrt{-3})$. This is a vector valued function on the quaternionic upper half space $H$. We have developed a vector version of the classical Rankin-Selberg integral used in [5], defined a $3 \times 3$ matrix 3 -cover Eisenstein series and studied the analytic properties, functional equations, poles and residues of the convolution.

In the quadratic case investigated by [5], the existence of infinitely many nonvanishing quadratic twists of the $L$-series is tied to the nonvanishing of certain residues. These residues are expressed in terms of a Rankin-Selberg convolution of $f$ with the quadratic theta function on $G L(2)$. In turn, these are simply the symmetric square $L$-functions of $f$. Their nonvanishing properties are well understood at the relevant point, which is at the border of the critical strip.

Unfortunately, in the cubic case the corresponding residues are convolutions of $F$ with the cubic theta function on $G L(2)$. This $L$-series does not have an Euler product, and hence its nonvanishing is considerably more subtle. After deriving the functional equation and analytic properties of the convolution, we will be able to describe an algorithm to prove that this convolution is non-zero at a certain point for any given form of $f$. Then we will be able to conclude that there are infinitely many cubic twists of $f$ which are non-zero at the center of the critical strip. The new form of weight 2 level 11 is used as an example to illustrate our method.

\section{Notation AND PRELIMINARIES}

We shall summarize a few basic facts about basis change of modular forms. The reference is S. Friedberg [6].

Let $f$ be a cusp form of weight 2 over $\mathbb{Q}$ for the congruence group $\Gamma_{0}(N)$ with Fourier expansion $f=\sum_{n=1}^{\infty} C(n) e^{2 \pi i n z}$, where $\Gamma_{0}(N)=\left\{\left(\begin{array}{ll}a & b \\ c & d\end{array}\right) \in S L(2, \mathbb{Z})|N| c\right\}$ and $N$ is a prime $\equiv 2(\bmod 3)$. Here $C(n)$ is the $n$th Fourier coefficient.

Let $K=\mathbb{Q}(\sqrt{-3}), O_{K}=\mathbb{Z}[\omega]\left(\omega=e^{\frac{2 \pi i}{3}}\right)$ be the integer ring of $\mathrm{K}$. The different of $K$ is the ideal $(\lambda)$, where $\lambda=\sqrt{-3}$. Let $H=\{z=x+y \vec{k} \mid x \in \mathbb{C}, y>0\}$, the quaternionic upper half space. Let $S L\left(2, O_{K}\right)$ act on $H$ in the usual way. If $x \in \mathbb{C}$, $x=u+i v$, let $e(x)=e^{4 \pi i u}$.

Following S. Friedberg [6], we have liftings of $f$ over $K$. Let $F=\left[\vec{F}_{\alpha}\right], \alpha=$ $1,0,-1$, be a lifting of $f$ on $H$. $F$ has a Fourier expansion as follows:

$$
\begin{aligned}
F & =\left[\begin{array}{c}
F_{1} \\
F_{0} \\
F_{-1}
\end{array}\right] \\
& =\sum_{m \in O_{K}} a_{m}\left[\begin{array}{c}
\frac{i}{2} \xi\left(\lambda^{-1} m\right) W_{1}\left(\left|\lambda^{-1} m\right| y\right) \\
W_{0}\left(\left|\lambda^{-1} m\right| y\right) \\
\frac{-i}{2} \xi^{-1}\left(\lambda^{-1} m\right) W_{1}\left(\left|\lambda^{-1} m\right| y\right)
\end{array}\right] e\left(\lambda^{-1} m x\right)
\end{aligned}
$$


where

$$
W_{\alpha}=y \cdot K_{\alpha}(y), \xi^{\alpha}(m)=\left(\frac{m}{|m|}\right)^{\alpha}
$$

and

$$
K_{\alpha}(y)=\frac{1}{2} \int_{0}^{\infty} e^{-\frac{y}{2}\left(t+t^{-1}\right)} t^{\alpha} \frac{d t}{t}
$$

is the standard $K$-Bessel function.

The coefficients $a_{m}$ are multiplicative and are given as follows:

$$
a\left(\wp^{n}\right)=(\mathcal{N} \wp)^{-n / 2} \begin{cases}C\left(p^{n}\right), & \chi_{-3}(p)=1, \mathcal{N} \wp=p \\ \sum_{f=0}^{n}(-1)^{f} p^{f} C\left(p^{2 n-2 f}\right), & \chi_{-3}(p)=-1, \wp=p O_{K} .\end{cases}
$$

In particular

$$
a(\wp)= \begin{cases}p^{-1 / 2} C(p), & \chi_{-3}(p)=1 \\ p^{-1}\left(C\left(p^{2}\right)-p\right), & \chi_{-3}(p)=-1 .\end{cases}
$$

It is easy to check

$$
\sum_{m \in O_{K} / O_{K}^{*}} \frac{a(m)(\mathcal{N} m)^{1 / 2}}{(\mathcal{N} m)^{s}}=\sum_{n=1}^{\infty} C(n) n^{-s} \sum_{n=1}^{\infty} C(n) \chi_{-3}(n) n^{-s} .
$$

For $g=\left(\begin{array}{l}a \\ c d \\ c\end{array}\right) \in S L(2, \mathbb{C}), \quad z=x+y \vec{k} \in H$; define:

$$
J_{3}(g, z)=\left(\begin{array}{ccc}
(c x+d)^{2} & -(c x+d) c y & (c y)^{2} \\
2(c x+d) \overline{c y} & |c x+d|^{2}-|c y|^{2} & -2 c y c x+d \\
\overline{c y}^{2} & \overline{(c x+d) c y} & \frac{c x+d}{c x+d}
\end{array}\right) .
$$

Let $\Lambda_{0}(N)=\left\{\left(\begin{array}{cc}a b \\ c d\end{array}\right) \in S L\left(2, O_{K}\right)|N| c, a \equiv d \equiv 1(\bmod 3)\right\}$. Let $\omega_{N}=\left(\begin{array}{cc}0 & -1 \\ N & 0\end{array}\right) \frac{1}{\sqrt{N}}$. Following S. Friedberg [6], page 8, we have, for $\gamma \in \Lambda_{0}(N)$,

$$
F(\gamma z)=J_{3}(\gamma, z) F(z)
$$

and

$$
F\left(\omega_{N} z\right)=J_{3}\left(\omega_{N}, z\right) F(z)
$$

Let $(\bullet)$ be the cubic residue symbol; for its basic properties see [9], page 112 . For $\mu \in\left(\lambda^{-3}\right), d \in O_{K}, d \equiv 1(\bmod 3)$, the cubic Gauss sum is defined by

$$
g(\mu, d)=\sum_{\delta(\bmod d)}\left(\frac{3 \delta}{d}\right) e\left(\frac{3 \mu \delta}{d}\right) .
$$

We summarise the basic properties of the cubic Gauss sum in the following proposition (see [4], page 486 for details).

Proposition 1.1. i) If $(a, d)=1$,

$$
g(a \mu, d)=\overline{\left(\frac{a}{d}\right)} g(\mu, d)=\left(\frac{a}{d}\right)^{2} g(\mu, d) .
$$


ii) If $\left(d, d^{\prime}\right)=1, d \equiv d^{\prime} \equiv 1(\bmod 3)$,

$$
g\left(\mu, d d^{\prime}\right)=\left(\frac{d}{d^{\prime}}\right)^{2} g(\mu, d) g\left(\mu, d^{\prime}\right)
$$

iii) If $p$ is prime, $p \equiv 1(3)$, then $|g(1, p)|=\sqrt{\mathcal{N} p}=|p|$,

$$
g\left(p^{k}, p^{l}\right)=\left\{\begin{array}{lc}
\mathcal{N} p^{k} \cdot g(1, p) & \text { if } l=k+1, k \equiv 0(3), \\
\mathcal{N} p^{k} \cdot \frac{g(1, p)}{} & \text { if } l=k+1, k \equiv 1(3), \\
-\mathcal{N} p^{k} & \text { if } l=k+1, k \equiv 2(3), \\
\mathcal{N} p^{l}-\mathcal{N} p^{l-1} & \text { if } l \equiv 0(3), k \geq l, \\
0 & \text { otherwise. }
\end{array}\right.
$$

The following L-series appears naturally in the Fourier coefficients of the metaplectic Eisenstein series. Define

$$
\Psi(s, \mu)=\sum_{c \equiv 1(3)} \frac{g(\mu, c)}{(\mathcal{N} c)^{s}} .
$$

The residue of $\Psi(s, \mu)$ at $s=4 / 3$ was established by Patterson [13]. Let us recall some specific results of Patterson [13], page 160, and of Bump and Hoffstein [4], page 487. Note that the function $\Psi(s, \mu)$ is $\Psi(s, \mu, 0)$ in [13].

Proposition 1.2. i)

$$
\underset{s=4 / 3}{\operatorname{Res}} \Psi(s, \mu)=C_{0} \frac{\tau(\mu)}{|\mu|^{1 / 3}}
$$

where

$$
\begin{gathered}
C_{0}=\frac{(2 \pi)^{2}}{3^{8} \cdot 2 \cdot \zeta(2)} \sigma \frac{V \cdot \Gamma\left(\frac{4}{3}\right)}{(2 \pi)^{4 / 3}}, \sigma=\sqrt{3^{5} / 4}, V=9 \cdot \frac{\sqrt{3}}{2} \\
\tau(\mu)= \begin{cases}\overline{g\left(\lambda^{2}, c\right)}\left|\frac{d}{c}\right| 3^{n / 2+2} & \text { if } \mu= \pm \lambda^{3 n-4} c d^{3}, n \geq 1, \\
e^{\frac{-2 \pi i}{9}} \frac{g\left(\omega \lambda^{2}, c\right)}{e^{2 \pi \pi}}\left|\frac{d}{c}\right| 3^{n / 2+2} & \text { if } \mu= \pm \omega \lambda^{3 n-4} c d^{3}, n \geq 1, \\
e^{\frac{2\left(\omega^{2} \lambda^{2}, c\right)}{g\left(\frac{d}{c}\right.} \mid 3^{n / 2+2}} & \text { if } \mu= \pm \omega^{2} \lambda^{3 n-4} c d^{3}, n \geq 1, \\
\hline(1, c)\left|\frac{d}{c}\right| 3^{(n+5) / 2} & \text { if } \mu= \pm \lambda^{3 n-3} c d^{3}, n \geq 0, \\
0 & \text { otherwise, }\end{cases}
\end{gathered}
$$

with $c \equiv d \equiv 1(\bmod 3), c, d \in O_{K}$ and $c$ square free.

ii) Define

$$
a(\mu)= \begin{cases}\overline{g(1, f)}\left|\frac{h}{f}\right| & \text { if } \mu=f h^{3}, f \equiv h \equiv 1(3), f \text { sq free, } \mu \in O_{K}, \\ 0 & \text { otherwise. }\end{cases}
$$

If $\left(\mu, \mu^{\prime}\right)=1, \mu \equiv 1(\bmod 3), \mu \in O_{K}, \mu^{\prime} \in\left(\lambda^{-3}\right)$, then we have

$$
\tau\left(\mu \mu^{\prime}\right)=\left(\frac{\mu^{\prime}}{\mu}\right) a(\mu) \tau\left(\mu^{\prime}\right) .
$$




\section{The Eisenstein Series And their expansion; Theta series}

In this section, we will define some metaplectic Eisenstein series and compute their residues at the relevant poles.

Let $\Lambda$ be the principal congruence subgroup modulo 3 of $S L\left(2, O_{K}\right)$. For $\gamma \in \Lambda$, we define the Kubota symbol

$$
\kappa(\gamma)= \begin{cases}1 & \text { if } c=0, \\ \left(\frac{c}{d}\right) & \text { if } c \neq 0,\end{cases}
$$

where $(\bullet)$ is the usual cubic residue symbol. Kubota observed that $\kappa$ is a character of $\Lambda$.

$$
\text { If } z=x+y \vec{k} \in H \text {, let } y=y(z) \text {. Define }
$$

$$
E_{\infty}(z, s)=\sum_{\gamma \in \Lambda_{\infty} \backslash \Lambda_{0}(N)}\left(\frac{N}{\pi(\gamma)}\right)^{2} \kappa(\gamma) y(\gamma z)^{2 s},
$$

where $\pi\left(\left(\begin{array}{ll}a & b \\ c & d\end{array}\right)\right)=d$ and $\Lambda_{\infty}=\left\{\gamma \in \Lambda_{0}(N) \mid \gamma(\infty)=\infty\right\}$. Then we have

$$
E_{\infty}(\gamma z, s)=\left(\frac{N}{\pi(\gamma)}\right) \overline{\kappa(\gamma)} E_{\infty}(z, s), \quad \forall \gamma \in \Lambda_{0}(N)
$$

Define

$$
E_{0}(z, s)=E_{\infty}\left(\omega_{N} z, s\right) \text {, i.e. } E_{\infty}\left(\frac{1}{\sqrt{N}}\left(\begin{array}{cc}
0 & -1 \\
N & 0
\end{array}\right) z, s\right) .
$$

Then it is easily checked that

$$
E_{0}(\gamma z, s)=\overline{\kappa(\gamma)} E_{0}(z, s), \quad \forall \gamma \in \Lambda_{0}(N) .
$$

As $E_{0}\left(z+3 O_{K}, s\right)=E_{0}(z, s), E_{0}(z, s)$ has a Fourier expansion

\section{Proposition 2.1.}

$$
\begin{aligned}
E_{0}(z, s)= & \sum_{m \in\left(\lambda^{-3}\right)} a_{m}(s, y) e(m x) \\
= & \frac{(2 \pi)^{2 s} N^{-2 s}}{\Gamma(2 s) V\left(\mathbb{C} / 3 O_{K}\right)} \sum_{\substack{m \in\left(\lambda^{-3}\right) \\
m \neq 0}} \frac{A_{m}(s) \mathcal{N}(m)^{s-1 / 2}}{\mathcal{N}(m)^{1 / 2}} W_{2 s-1}(|m| y) e(m x) \\
& +a_{0}(s, y)
\end{aligned}
$$

where

$$
\begin{aligned}
a_{0}(s, y) & =y^{2-2 s} \frac{\pi}{2 s-1} \frac{\zeta_{N}(6 s-3)}{\zeta_{N}(6 s-2)}, \\
\zeta_{N}(s) & =\left(1-\mathcal{N} N^{-s}\right) \zeta(s) \\
A_{m}(s) & =\sum_{\substack{d \equiv 1(3) \\
(d, N)=1}} \frac{g(m, d)}{(\mathcal{N} d)^{2 s}}, \zeta(s)=\sum_{d \equiv 1(3)} \frac{1}{(\mathcal{N} d)^{s}},
\end{aligned}
$$

and $V\left(\mathbb{C} / 3 O_{K}\right)$ is the Euclidean volume of $\mathbb{C} / 3 O_{K}$.

It is clear from the term $a_{0}(s, y)$ that $E_{0}(z, s)$ has a simple pole at $s=2 / 3$. The residue can be taken out at this point yielding the cubic theta function. 
Define the theta series,

$$
\begin{aligned}
\theta(z) & =\operatorname{Res}_{s=2 / 3} E_{0}(z, s) \\
& =C_{2} \sum_{\substack{m \in\left(\lambda^{-3}\right) \\
m \neq 0}} \frac{\operatorname{Res}_{s=2 / 3} A_{m}(s) \mathcal{N}(m)^{1 / 6}}{\mathcal{N}(m)^{1 / 2}} W_{1 / 3}(|m| y) e(m x)+C y^{2 / 3} \\
& =C_{1} \sum_{\substack{m \in\left(\lambda^{-3}\right) \\
m \neq 0}} \frac{\tau(m)}{\mathcal{N}(m)^{1 / 2}} W_{1 / 3}(|m| y) e(m x)+C y^{2 / 3}
\end{aligned}
$$

where

$$
C_{2}=\frac{(2 \pi)^{4 / 3} N^{-4 / 3}}{\Gamma(4 / 3) V\left(\mathbb{C} / 3 O_{K}\right)}, C_{1}=\frac{1}{2} \cdot C_{2} C_{0} \frac{1-N^{-2}}{1-N^{-4}}
$$

and $C$ is a constant which we shall not need.

The coefficients $\tau(m)$ are those of Patterson's theta function and are described in (1.10). In (2.7) we make use of the following lemma.

Lemma 2.2. For $m \in\left(\lambda^{-3}\right)$, we have

$$
\operatorname{Res}_{s=4 / 3} \sum_{\substack{d \equiv 1(3) \\(d, N)=1}} \frac{g(m, d)}{(\mathcal{N} d)^{s}}=C_{0} \frac{\tau(m)}{|m|^{1 / 3}} \cdot \frac{1-N^{-2}}{1-N^{-4}}
$$

Note that the residue in (2.8) has an extra condition $(d, N)=1$ when compared to Patterson's result (1.9).

Proof of Lemma 2.2. For $m \in\left(\lambda^{-3}\right)$, define

$$
\begin{aligned}
\tilde{A}_{m}(s) & =\sum_{\substack{d \equiv 1(3) \\
(d, N)=1}} \frac{g(m, d)}{\mathcal{N} d^{s}}, \tilde{B}_{m}(s)=\sum_{\substack{d \equiv 1(3) \\
(d, N)=1}} \frac{g\left(N^{2} m, d\right)}{\mathcal{N} d^{s}} \\
\tilde{C}_{m}(s) & =\sum_{\substack{d \equiv 1(3) \\
(d, N)=1}} \frac{g(N m, d)}{\mathcal{N} d^{s}}
\end{aligned}
$$

Set

$$
\begin{gathered}
u_{m}=\operatorname{Res}_{s=4 / 3} \tilde{A}_{m}(s)=2 \cdot \operatorname{Res}_{s=2 / 3} A_{m}(s), \\
v_{m}=\operatorname{Res}_{s=4 / 3} \tilde{B}_{m}(s), w_{m}=\operatorname{Res}_{s=4 / 3} \tilde{C}_{m}(s) .
\end{gathered}
$$


To compute $v_{m},(m, N)=1$, we start with

$$
\begin{aligned}
& 0=C_{0} \frac{\tau\left(N^{2} m\right)}{\left|N^{2} m\right|^{1 / 3}}=\operatorname{Res}_{s=4 / 3} \sum_{d \equiv 1(3)} \frac{g\left(N^{2} m, d\right)}{\mathcal{N} d^{s}} \\
& =\operatorname{Res}_{s=4 / 3}\left(\sum_{\substack{d \equiv 1(3) \\
(d, N)=1}} \frac{g\left(N^{2} m, d\right)}{\mathcal{N} d^{s}}+\sum_{\substack{d \equiv 1(3) \\
(d, N)=1}} \frac{g\left(N^{2} m, N^{3} d\right)}{\mathcal{N} d^{s}}\right) \\
& =v_{m}+\operatorname{Res}_{s=4 / 3} \frac{g\left(N^{2} m, N^{3}\right)}{\left(\mathcal{N} N^{3}\right)^{s}} \cdot \sum_{\substack{d \equiv 1(3) \\
(d, N)=1}} \frac{g\left(N^{2} m, d\right)}{\mathcal{N} d^{s}} \\
& =v_{m}+\frac{-(\mathcal{N N})^{2}}{(\mathcal{N N})^{4}} v_{m} \\
& =\left(1-N^{-4}\right) v_{m} \text {. }
\end{aligned}
$$

Thus

$$
v_{m}=0 .
$$

Using a similar technique for $u_{m}, w_{m}$, with $(m, N)=1$, we have a linear system

$$
\left\{\begin{aligned}
u_{m}+\left(\frac{m}{N}\right)^{2} g(1, N) N^{-8 / 3} w_{m} & =C_{0} \frac{\tau(m)}{|m|^{1 / 3}}, \\
\left(\frac{m}{N}\right) \overline{g(1, N)} N^{-10 / 3} u_{m}+w_{m} & =C_{0} \frac{\tau(m)}{|m|^{1 / 3}} \\
& =\frac{\frac{\mid(1, N)}{\left(1, \frac{m}{N}\right)}}{N^{4 / 3}} C_{0} \frac{\tau(N m)}{N|m|^{1 / 3}} .
\end{aligned}\right.
$$

Solving this linear system, we have proved $(2.8)$ for $(m, N)=1$. We have

$$
u_{m}=C_{0} \frac{\tau(m)}{|m|^{1 / 3}} \frac{1-N^{-2}}{1-N^{-4}}
$$

and

$$
w_{m}=C_{0} \frac{\tau(m)}{|m|^{1 / 3}} \frac{N^{4 / 3}\left(1-N^{-2}\right)\left(\frac{m}{N}\right) \overline{g(1, N)}}{1-N^{-4}} .
$$

Now write $m=N^{j} m^{\prime}$, with $\left(m^{\prime}, N\right)=1$.

Then in each of the three cases $j \equiv 0,1,2(\bmod 3)$, we write the answer in terms of $m^{\prime}$ first, using the previous result for $\left(m^{\prime}, N\right)=1$, then write the answer in terms of $m$. We find the answers are the same as the right side of (2.8) in terms of $m$ for all cases. This completes the proof of Lemma 2.2.

Using the multiplier $J_{3}(g, z)$, we can define a Eisenstein series:

$$
E_{\infty}^{3}(z, w)=\sum_{\gamma \in \Lambda_{\infty} \backslash \Lambda_{0}(N)} \kappa(\gamma) J_{3}(\gamma, z) y^{2 s}(\gamma z) .
$$

This is a $3 \times 3$ matrix function satisfying

$$
E_{\infty}^{3}(\delta z, s)=E_{\infty}^{3}(z, s) \overline{\kappa(\delta)} J_{3}^{-1}(\delta, z), \quad \forall \delta \in \Lambda_{0}(N) .
$$


We will need the expansion of $E_{\infty}^{3}(z, s)$ at the cusp 0 . We define

$$
\begin{aligned}
E_{0}^{3}(z, s) & =E_{\infty}^{3}\left(\omega_{N} z, s\right) J_{3}\left(\omega_{N}, z\right) \\
& =\sum_{\gamma \in \Lambda_{\infty} \backslash \Lambda_{0}(N)} \kappa(\gamma) J_{3}\left(\gamma \omega_{N}, z\right) y^{2 s}\left(\gamma \omega_{N} z\right) .
\end{aligned}
$$

It transforms as follows:

$$
E_{0}^{3}(\delta z, s)=E_{0}^{3}(z, s)\left(\frac{N}{\pi(\delta)}\right) \overline{\kappa(\delta)} J_{3}^{-1}(\delta, z) .
$$

It is easy to check that $E_{0}^{3}\left(z+3 O_{K}, s\right)=E_{0}^{3}(z, s)$. Thus $E_{0}^{3}(z, s)$ has the Fourier expansion

\section{Proposition 2.3.}

$$
E_{0}^{3}(z, s)=\sum_{m \in\left(\lambda^{-3}\right)} a_{m}^{3}(s, y) e(m x) .
$$

Then, for $m \neq 0$

$$
\begin{aligned}
& a_{m}^{3}(s, y)=y^{2 s} N^{1-2 s} y^{4-4 s} \frac{1}{V\left(\mathbb{C} / 3 O_{K}\right)} \sum_{\substack{d \equiv 1(3) \\
(d, N)=1}}\left(\begin{array}{lll}
\left(\frac{d}{|d|}\right)^{2} & & \\
& 1 & \\
& & \left(\frac{\bar{d}}{|d|}\right)^{2}
\end{array}\right) \\
& \times \frac{\left(\frac{N}{d}\right) g(m, d)}{(\mathcal{N} d)^{2 s-1}} \int_{\mathbb{C}}\left(\begin{array}{ccc}
x^{2} & -x & 1 \\
2 x & |x|^{2}-1 & -2 \bar{x} \\
1 & \bar{x} & \bar{x}^{2}
\end{array}\right) \frac{\overline{e(m x)}}{\left(|x|^{2}+1\right)^{2 s}} d x .
\end{aligned}
$$

When $m=0$, we have

$$
J \cdot a_{0}^{3}(s, y)=\frac{\zeta_{N}(6 s-6)}{\zeta_{N}(6 s-5)} \cdot \frac{\pi}{2 s-2} \cdot \frac{3-2 s}{2 s-1} \cdot y^{4-2 s},
$$

where $\zeta_{N}(s)$ is as in (2.6), $J=[0,1,0]$.

We can see that $E_{0}^{3}(z, s)$ has a pole at $s=7 / 6$. Let us define a new theta series

$$
\Theta^{3}(z)=\operatorname{Res}_{s=7 / 6} J \cdot E_{0}^{3}(z, s),
$$

which is a 3-dimension row vector. Assembling the above information from (2.8) and (2.20), we have 


\section{Proposition 2.4.}

$$
\begin{aligned}
& \Theta^{3}(z)=\tilde{C} y^{5 / 3}+\frac{y^{5 / 3} N^{-8 / 6}}{V\left(\mathbb{C} / 3 O_{K}\right)} \sum_{\substack{m \in\left(\lambda^{-3}\right) \\
m \neq 0}} \operatorname{Res}_{s=7 / 6} \sum_{\substack{d \equiv 1(3) \\
(d, N)=1}} \frac{g\left(N^{2} m, d\right)}{(\mathcal{N} d)^{2 s-1}} \cdot e(m x) \\
& \cdot\left(\begin{array}{c}
2 i^{-1} \frac{(2 \pi)^{7 / 3}}{\Gamma(7 / 3)} \xi^{-1}(m)|m y|^{4 / 3} K_{1 / 3}(4 \pi|m y|) \\
\frac{(2 \pi)^{4 / 3}}{\Gamma(4 / 3)}|m y|^{1 / 3} K_{1 / 3}(4 \pi|m y|)-2 \frac{(2 \pi)^{7 / 3}}{\Gamma(7 / 3)}|m y|^{4 / 3} K_{4 / 3}(4 \pi|m y|) \\
-2 i^{-1} \frac{(2 \pi)^{7 / 3}}{\Gamma(7 / 3)} \xi(m)|m y|^{4 / 3} K_{1 / 3}(4 \pi|m y|)
\end{array}\right)^{t} \\
& =\tilde{C} y^{5 / 3}+\frac{N^{-4 / 3} \cdot(2 \pi)^{4 / 3} C_{0}}{2 \cdot V\left(\mathbb{C} / 3 O_{K}\right) \Gamma(4 / 3)} \cdot \frac{1-N^{-2}}{1-N^{-4}} \sum_{\substack{m \in\left(\lambda^{-3}\right) \\
m \neq 0}} \frac{\tau\left(N^{2} m\right)}{\left|N^{2} m\right|^{1 / 3}} \text {. } \\
& \times y^{5 / 3} \cdot\left(\begin{array}{c}
-3 i \pi \xi^{-1}(m)|m y|^{1 / 3} W_{1 / 3}(|m y|) \\
|m y|^{-2 / 3} W_{1 / 3}(|m y|)-3 \pi|m y|^{1 / 3} W_{4 / 3}(|m y|) \\
3 i \pi \xi(|m|)|m y|^{1 / 3} W_{1 / 3}(|m y|)
\end{array}\right)^{t} e(m x)
\end{aligned}
$$

where $\tilde{C}$ is a constant which we shall not need.

\section{Convolutions And their Residues}

In this section, we will define a convolution of $F(z)$ with a metaplectic Eisenstein series. The convolution may be written as a product of a Dirichlet series with a convolution of Whittaker functions. Using the technique of [5], we may express the Dirichlet series as a sum of weighted cubic twists $L\left(s, F \otimes\left(\frac{\bullet}{d}\right)\right)$. Further we compute the residue of the convolution at the relevant poles.

Define a convolution as follows:

$$
R(F, s, w)=\left[\begin{array}{c}
R_{1} \\
R_{0} \\
R_{-1}
\end{array}\right]=\iint_{D} E_{\infty}^{3}(z, w+s) F(z) \overline{E_{0}(z, \bar{s})} \frac{d x d y}{y^{3}}
$$

where $D=\Lambda_{0}(N) \backslash H$.

This is a well defined 3 -dimensional integral. As one can easily verify, $y^{-3} d x d y$ is an invariant volume element, and the integrand is invariant under the action of $z \longrightarrow \gamma z$, for any $\gamma \in \Lambda_{0}(N)$. Furthermore the integral converges if Re $w$, Re $s$ are sufficiently large. This is because the Eisenstein series converges absolutely for those values of $w$ and $s$.

Substituting for the Fourier expansion of $F(z), E_{0}(z, s)$ from (1.1) and (2.5) and "unfolding", we have

$$
\begin{aligned}
R_{0}(F, s, w)= & \frac{(2 \pi)^{2 s} N^{-2 s}}{\Gamma(2 s) V\left(\mathbb{C} / 3 O_{K}\right) 3^{-w}} \sum_{m \in O_{K}} \frac{a_{m} \overline{A_{\lambda^{-1} m}}(\bar{s})}{(\mathcal{N} m)^{w}} \\
& \times \int_{0}^{\infty} y^{2(w+s)-2} W_{0}(y) \overline{W_{2 s-1}(y)} \frac{d y}{y} \\
= & L(s, w) \cdot G(s, w),
\end{aligned}
$$


where

$$
\begin{gathered}
L(s, w)=\sum_{m \in O_{K}} \frac{a_{m} \overline{A_{\lambda^{-1} m}}(\bar{s})}{(\mathcal{N} m)^{w}}, \\
G(s, w)=\frac{(2 \pi)^{2 s} N^{-2 s} 3^{w}}{\Gamma(2 s) V\left(\mathbb{C} / 3 O_{K}\right)} \int_{0}^{\infty} y^{2(w+s)-2} W_{0}(y) \overline{W_{2 s-1}(y)} \frac{d y}{y},
\end{gathered}
$$

and both $L(s, w), G(s, w)$ converge if $\operatorname{Re} w$, Re $s$ are sufficiently large.

We now apply the technique of [5] and interchange the order of summation to rewrite $L(s, w)$ :

$$
\begin{aligned}
L(s, w) & =\sum_{m \in O_{K}} \frac{a_{m}}{(\mathcal{N} m)^{w}} \sum_{\substack{d \equiv 1(3) \\
(d, N)=1}} \frac{\overline{g\left(\lambda^{-1} m, d\right)}}{(\mathcal{N} d)^{2 s}} \\
& =\sum_{\substack{d \equiv 1(3) \\
(d, N)=1}} \frac{1}{(\mathcal{N} d)^{2 s}}\left(\sum_{m \in O_{K}} \frac{a_{m} \overline{g\left(\lambda^{-1} m, d\right)}}{(\mathcal{N} m)^{w}}\right) \\
= & \sum_{\substack{d \equiv 1(3) \\
(d, N)=1}} \frac{1}{(\mathcal{N} d)^{2 s}}\left(\sum_{\substack{p|m \\
\Rightarrow p| d}} \frac{a_{m} \overline{g\left(\lambda^{-1} m, d\right)}}{(\mathcal{N} m)^{w}}\right)\left(\sum_{\substack{(m, d)=1 \\
m \in O_{K}}} \frac{\left(\frac{m}{d}\right) a_{m}}{(\mathcal{N} m)^{w}}\right) \\
= & \sum_{\substack{d \equiv 1(3) \\
(d, N)=1}} \frac{1}{(\mathcal{N} d)^{2 s}} B(d) L\left(w, F \otimes\left(\frac{\bullet}{d}\right)\right),
\end{aligned}
$$

where

$$
\begin{aligned}
B(d) & =\left(\sum_{\substack{p|m \\
\Rightarrow p| d \\
m \in O_{K}}} \frac{a_{m} \overline{g\left(\lambda^{-1} m, d\right)}}{(\mathcal{N} m)^{w}}\right), \\
L\left(w, F \otimes\left(\frac{\bullet}{d}\right)\right) & =\sum_{m \in O_{K}} \frac{a_{m}\left(\frac{m}{d}\right)}{(\mathcal{N} m)^{w}} .
\end{aligned}
$$

Here $p|m \Rightarrow p| d$ means that every prime factor of $m$ is a prime factor of $d$.

To compute $B(d)$, write $d=M d_{1}^{3},\left(M, d_{1}\right)=1$, that is,

$$
M=\prod_{\substack{p \mid d \\ \operatorname{ord}_{p} d \not 0(3)}} p^{\operatorname{ord}_{p} d}, d_{1}=\prod_{\substack{p \mid d \\ \operatorname{ord}_{p} d \equiv 0(3)}} p^{\frac{\operatorname{ord}_{p} d}{3}} .
$$

Let Supp $(q)=\{p$ prime $|p| q\}$; then we have that $\operatorname{Supp}(d)=\operatorname{Supp}(M) \cup$ $\operatorname{Supp}\left(d_{1}\right)$ and $\operatorname{Supp}(M) \cap \operatorname{Supp}\left(d_{1}\right)=\varnothing,(\varnothing$ is the empty set).

For $m \in O_{K}$, Supp $(m) \subseteq \operatorname{Supp}(d)$, write $m=m_{1} m_{2}$, with $\operatorname{Supp}\left(m_{1}\right) \subseteq$ $\operatorname{Supp}(M)$ and Supp $\left(m_{2}\right) \subseteq \operatorname{Supp}\left(d_{1}\right)$, i.e. $\left(m_{2}, M\right)=1,\left(m_{1}, d_{1}\right)=1$. 
Then we have,

$$
\begin{aligned}
g\left(\lambda^{-1} m, d\right) & =g\left(\lambda^{-1} m_{1} m_{2}, M d_{1}^{3}\right) \\
& =\left(\frac{M}{d_{1}^{3}}\right)^{2} g\left(\lambda^{-1} m_{1} m_{2}, M\right) g\left(\lambda^{-1} m_{1} m_{2}, d_{1}^{3}\right) \\
& =\left(\frac{m_{2}}{M}\right)^{2} g\left(m_{2}, d_{1}^{3}\right) g\left(\lambda^{-1} m_{1}, M\right) .
\end{aligned}
$$

As $a_{m}$ is multiplicative, we have

$$
B(d)=\sum_{\substack{\operatorname{Supp}\left(m_{2}\right) \\ \subseteq \operatorname{Supp}\left(d_{1}\right)}} \frac{\left(\frac{m_{2}}{M}\right) \overline{g\left(m_{2}, d_{1}^{3}\right)} a_{m_{2}}}{\left(\mathcal{N} m_{2}\right)^{w}} \sum_{\substack{\operatorname{Supp}\left(m_{1}\right) \\ \subseteq \operatorname{Supp}(M)}} \frac{\overline{g\left(\lambda^{-1} m_{1}, M\right)} a_{m_{1}}}{\left(\mathcal{N} m_{1}\right)^{w}} .
$$

Now, it is easily checked that the first summation in (3.8) is multiplicative. We have

$$
B(d)=\left(\prod_{p^{t} \| d_{1}^{3}} D_{t}(p)\right) \cdot D_{M}
$$

where

$$
\begin{aligned}
& D_{t}(p)=\sum_{r=0}^{\infty} \frac{\left(\frac{p^{r}}{M}\right) \overline{g\left(p^{r}, p^{t}\right)} a_{p^{r}}}{\left(\mathcal{N} p^{r}\right)^{w}} \\
& =\frac{\phi\left(p^{t}\right)(\mathcal{N} p)^{-t w}\left[a_{p^{t}}-(\mathcal{N} p)^{-w}\left(\frac{p}{M}\right) a_{p^{t-1}}\right]}{\left(1-\left(\frac{p}{M}\right) a_{p}(\mathcal{N} p)^{-w}+\left(\frac{p}{M}\right)^{2}(\mathcal{N} p)^{-2 w}\right)} \\
& -\left(\frac{p}{M}\right)^{2}(\mathcal{N} p)^{t-1} a_{p^{t-1}}(\mathcal{N} p)^{-(t-1) w}, \\
& D_{M}=\sum_{\substack{\operatorname{Supp}\left(m_{1}\right) \\
\subseteq \operatorname{Supp}(M)}} \frac{\overline{g\left(\lambda^{-1} m_{1}, M\right)} a_{m_{1}}}{\left(\mathcal{N} m_{1}\right)^{w}} \\
& =\frac{\overline{g\left(\lambda^{-1} M_{0}, M\right)} a_{M_{0}}}{\left(\mathcal{N} M_{0}\right)^{w}},
\end{aligned}
$$

with

$$
M_{0}=\prod_{p \mid M} p^{\operatorname{ord}_{p} M-1}, \quad \phi\left(p^{t}\right)=\mathcal{N} p^{t}-\mathcal{N} p^{t-1} .
$$

Thus, we have

Proposition 3.1.

$$
\begin{aligned}
L(s, w)= & \sum_{\substack{d \equiv 1(3) \\
(d, N)=1}} \frac{1}{(\mathcal{N} d)^{2 s}} B(d) L\left(w, F \otimes\left(\frac{\bullet}{d}\right)\right) \\
= & \sum_{\substack{d \equiv 1(3) \\
\left(M, d_{1}\right)=1,\left(M d_{1}, N\right) \\
p \mid M \Rightarrow \operatorname{ord}_{p} M \not \equiv 0(3)}} \frac{1}{\left(\mathcal{N} M d_{1}^{3}\right)^{2 s}} D_{M} \cdot \prod_{p^{t} \| d_{1}^{3}} \tilde{D}_{t}(p) \cdot L\left(w, F \otimes\left(\frac{\bullet}{M}\right)\right),
\end{aligned}
$$


where $\tilde{D}_{t}(p)$ and $D_{M}$ are both functions of $w$ and

$$
\tilde{D}_{t}(p)=D_{t}(p)\left(1-\left(\frac{p}{M}\right) a_{p}(\mathcal{N} p)^{-w}+\left(\frac{p}{M}\right)^{2}(\mathcal{N} p)^{-2 w}\right) .
$$

Let us compute the residues of the convolution $R(F, s, w)$. Recall that $E_{0}(z, s)$ has a pole at $s=2 / 3$, and its residue is $\theta(z)$, as computed in (2.7). Thus $R(F, s, w)$ has a pole at $s=2 / 3$. Let $R_{0}(F, s, w)=J \cdot R(F, s, w)$, with $J=\left[\begin{array}{lll}0 & 1 & 0\end{array}\right]$. Then, we have

\section{Proposition 3.2.}

$$
\underset{s=2 / 3}{\operatorname{Res}} R_{0}(F, s, w)=L(w+1 / 6) \cdot G(w+1 / 6)
$$

where

$$
L(s)=\sum_{m \in O_{K}} \frac{a_{m} \overline{\tau\left(\lambda^{-1} m\right)}}{(\mathcal{N} m)^{s}},
$$

the $a_{m}$ are the coefficients of $F$ (see (1.2)), the $\tau\left(\lambda^{-1} m\right)$ are coefficients of $\theta(z)$, as given in (1.10), and

$$
G(s)=C_{1} \cdot 3^{s} \cdot(4 \pi)^{-2(s+1)} \cdot 2^{2 s-2} \cdot \frac{\Gamma^{2}(s+1 / 3) \Gamma^{2}(s+2 / 3)}{\Gamma(2 s+1)} .
$$

Proof. Using the "unfolding" trick and recalling (1.1), (2.7), we have

$$
\begin{aligned}
\operatorname{Res}_{s=2 / 3} R_{0}(F, s, w)= & C_{1} \int_{0}^{\infty} \int_{\mathbb{C} / 3} y^{2(w+2 / 3)}\left(\sum_{m \in O_{K}} a_{m} W_{0}\left(\left|\lambda^{-1} m\right| y\right) e\left(\lambda^{-1} m x\right)\right) . \\
& \left(\sum_{m \in\left(\lambda^{-3}\right)} \frac{\overline{\tau(m)}}{(\mathcal{N} m)^{1 / 2}} W_{1 / 3}(|m| y) \overline{e(m x)}\right) \frac{d x d y}{y^{3}} \\
= & C_{1} \int_{0}^{\infty} \sum_{m \in O_{K}} \frac{a_{m} \overline{\tau\left(\lambda^{-1} m\right)}}{\left(\mathcal{N} \lambda^{-1} m\right)^{w+1 / 6}} y^{2(w-1 / 3)} W_{0}(y) W_{1 / 3}(y) \frac{d y}{y} \\
= & L(w+1 / 6) \cdot G(w+1 / 6) .
\end{aligned}
$$

Note that (cf. I. Gradshteyn and I. Ryzhik [8], page 716 (6.576) and page 1068 $(9.122))$,

$$
C_{1} 3^{s} \int_{0}^{\infty} y^{2(s-1 / 2)} W_{0}(y) W_{1 / 3}(y) \frac{d y}{y}=G(s) .
$$

This completes the proof of the proposition. 
To find the other residue of $R(F, s, w)$, first changing variables $z \rightarrow \omega_{N} z$, we have

$$
\begin{aligned}
R(F, s, w) & =\iint_{D} E_{\infty}^{3}(z, w+s) F(z) \overline{E_{0}(z, \bar{s})} \frac{d x d y}{y^{3}} \\
& =\iint_{D} E_{0}^{3}(z, w+s) F(z) \overline{E_{\infty}(z, \bar{s})} \frac{d x d y}{y^{3}} \\
& =\int_{0}^{\infty} \int_{\mathbb{C} / 3} E_{0}^{3}(z, w+s) F(z) y^{2 s} \frac{d x d y}{y^{3}} .
\end{aligned}
$$

Now, we know that $E_{0}^{3}(z, s)$ has a pole at $s=7 / 6$. Its residue is computed in Proposition 2.4. Thus $R(F, s, w)$ has a pole at $s=7 / 6-w$. We have

\section{Proposition 3.3.}

$$
\operatorname{Res}_{s=7 / 6-w} R_{0}(F, s, w)=(-1) \cdot G(7 / 6-w) \cdot \tilde{L}(7 / 6-w) \cdot \frac{3}{4} \cdot(7 / 6-2 w)
$$

where $\tilde{L}(s)=N^{-2 / 3} \sum_{m \in O_{K}} \frac{\tau\left(\lambda^{-1} N^{2} m\right) a_{m}}{(\mathcal{N} m)^{s}}$.

Proof. By Proposition 2.4 and (1.1), substituting Fourier expansion of $F(z)$ and $\Theta^{3}(z)$, we have

$$
\begin{aligned}
\operatorname{Res}_{s=7 / 6-w} R_{0}(F, s, w) \\
=\frac{N^{-4 / 3} \cdot(2 \pi)^{4 / 3} C_{0}}{2 \cdot V\left(\mathbb{C} / 3 O_{K}\right) \Gamma(4 / 3)} \cdot \frac{1-N^{-2}}{1-N^{-4}} \sum_{m \in\left(\lambda^{-1}\right)} \frac{\tau\left(N^{2} m\right) a_{\lambda m}}{\left|N^{2} m\right|^{1 / 3}} \\
\quad \times \int_{0}^{\infty}\left[|m y|^{-2 / 3} W_{0}(|m y|) W_{1 / 3}(|m y|)-3 \pi|m y|^{1 / 3} W_{0}(|m y|) W_{4 / 3}(|m y|)\right. \\
\left.-3 \pi|m y|^{1 / 3} W_{1}(|m y|) W_{1 / 3}(|m y|)\right] y^{4-2 w} \frac{d y}{y^{3}} \\
=C_{1}(4 \pi)^{2 w-\frac{10}{3}} \cdot 2^{-2 w+1 / 3} \cdot(-1) \frac{3}{4} \frac{\Gamma^{2}\left(\frac{7}{6}-w\right) \Gamma^{2}\left(\frac{3}{2}-w\right)}{\Gamma\left(\frac{7}{3}-2 w\right)}(\sqrt{3})^{7 / 2-w} \\
\quad \times \sum_{m \in O_{K}} \frac{\tau\left(\lambda^{-1} N^{2} m\right) a_{m}}{(\mathcal{N} m)^{7 / 6-w}} \cdot N^{\frac{-2}{3}} \\
=(-1) G(7 / 6-w) \cdot \tilde{L}(7 / 6-w) \frac{3}{4}(7 / 3-2 w),
\end{aligned}
$$

where

$$
\tilde{L}(s)=N^{-2 / 3} \sum_{m \in O_{K}} \frac{\tau\left(\lambda^{-1} N^{2} m\right) a_{m}}{(\mathcal{N} m)^{s}} .
$$

\section{The Functional equations}

The purpose of this section is to find the functional equations for $L(s)$. We start with computing the functional equations for the Eisenstein series defined in Section 2. 
Let $p$ be a cusp of $\Lambda_{0}(N), \Lambda_{p}=\left\{\gamma \in \Lambda_{0}(N) \mid \gamma(p)=p\right\}$. A cusp is called essential if the restriction of the Kubota map $\kappa$ to $\Lambda_{p}$ is trivial. It is easily checked that $\Lambda_{0}(N)$ has eight essential cusps, $\left\{\infty, 0,1,-1, \frac{1}{3}, \frac{3}{N},-\frac{1}{N}, \frac{1}{N}\right\}$. If $p$ is an essential cusp, then there is $\sigma_{p} \in S L(2, \mathbb{R})$, such that $\sigma_{p}(\infty)=p$, and $\sigma_{p} \Lambda_{\infty} \sigma_{p}^{-1}=\Lambda_{p}, \sigma_{p} \Lambda \sigma_{p}^{-1}=\Lambda$.

We may define the Eisenstein series at a essential cusp as follows:

$$
E_{p}^{3}(z, s)=\sum_{\gamma \in \Lambda_{\infty} \backslash \Lambda_{0}(N)} \kappa(\gamma) J_{3}\left(\gamma \sigma_{p}^{-1}, z\right) y^{2 s}\left(\gamma \sigma_{p}^{-1} z\right) .
$$

Let us put all $J \cdot E_{p}^{3}(z, s)$ in the same order as in $\left\{\infty, 0,1,-1, \frac{1}{3}, \frac{3}{N},-\frac{1}{N}, \frac{1}{N}\right\}$ to form a eight dimensional vector $\overrightarrow{E^{3}}(z, s)$. That is,

$$
\overrightarrow{E^{3}}(z, s)=\left[\begin{array}{c}
\vdots \\
J \cdot E_{p}^{3}(z, s) \\
\vdots
\end{array}\right]_{8 \times 1} .
$$

In particular the entry on the top is $E_{\infty}^{3}(z, s)$.

For this vector $\vec{E}^{3}(z, s)$, we have a functional equation as follows:

\section{Proposition 4.1.}

$$
\overrightarrow{E^{3}}(z, s)=\Phi(s) \cdot \overrightarrow{E^{3}}(z, 2-s) \cdot V^{-1} \cdot \frac{3^{6 s-5}-1}{3^{6 s-6}-1} \cdot \frac{\zeta(6 s-6)}{\zeta(6 s-5)} \cdot \frac{\pi}{2 s-2} \cdot \frac{3-2 s}{2 s-1},
$$

where

$$
\begin{aligned}
& \Phi(s)=\left[a_{i j}(s)\right]_{8 \times 8}=\left(\frac{3^{6 s-5}-1}{3^{6 s-6}-1}\right)^{-1}\left(\begin{array}{cccc}
\frac{2}{3^{6 s-6}-1} & 1 & 1 & 1 \\
1 & \frac{2}{3^{6 s-6}-1} & 1 & 1 \\
1 & 1 & \frac{2}{3^{6 s-6}-1} & 1 \\
1 & 1 & 1 & \frac{2}{3^{6 s-6}-1}
\end{array}\right) \\
& \otimes\left(\begin{array}{ll}
A_{N} & B_{N} \\
B_{N} & C_{N}
\end{array}\right) .
\end{aligned}
$$

Here $\otimes$ is the Kronecker product and

$$
\begin{aligned}
A_{N} & =\frac{\left(N^{2}-1\right) N^{10-12 s}}{1-N^{10-12 s}}, \\
B_{N} & =N^{1-2 s} \frac{1-N^{12-12 s}}{1-N^{10-12 s}}, \\
C_{N} & =\frac{\left(N^{2}-1\right) N^{2-4 s}}{1-N^{10-12 s}} .
\end{aligned}
$$

Proof. For reasons of space we omit this easy but lengthy computation. Basically, one gets the scattering matrices from the constant terms of the Fourier expansions of the Eisenstein series at the various cusps. A similar computation can be found in [13].

We will be using the functional equation of $L(s)$ in Section 5 to compute $L(2 / 3)$ under the assumption that $L(s)$ has no pole at $s=2 / 3$. Here we are going to find the exact functional equation of $L(s)$. Let $J$ be the row vector $\left[\begin{array}{lll}0 & 1 & 0\end{array}\right]$. Then, 
$J \cdot R(F, s, w)=R_{0}(F, s, w)$, and the residue of $R_{0}(F, s, w)$ involves $L(s)$. Referring to Proposition 3.2, we have

$$
\begin{aligned}
L(s) G(s)= & J \cdot \iint_{D} E_{\infty}^{3}(z, s+1 / 2) F(z) \overline{\theta(z)} \frac{d x d y}{y^{3}} \\
= & J \cdot \iint_{D} \sum_{j=1}^{8} a_{1 j}(s+1 / 2) E_{j}(z,(1-s)+1 / 2) F(z) \overline{\theta(z)} \frac{d x d y}{y^{3}} \\
= & \sum_{j=1}^{8} a_{1 j}(s+1 / 2) \cdot J \cdot \iint_{D} E_{j}(z,(1-s)+1 / 2) F(z) \overline{\theta(z)} \frac{d x d y}{y^{3}} \\
= & \sum_{j=1}^{8} a_{1 j}(s+1 / 2) a_{2 j}(7 / 6) L_{j}(1-s) G(1-s) \\
& \quad \times \frac{\zeta^{\star}(6(1-s)-2)}{\zeta^{\star}(6 s-2)} \cdot \frac{1-s}{s} \cdot 3^{3-6 s},
\end{aligned}
$$

where

$$
L_{j}(s)=\sum_{m \in O_{K}} \frac{a_{m}^{j} \overline{\tau\left(\lambda^{-1} m\right)}}{(N m)^{s}} .
$$

In the above $a_{m}^{j}$ is the Fourier coefficient of $F(z)$ expanded at the cusp $j$. In particular $L_{\infty}(s)=L(s)$, and the $a_{i j}(s)$ are the entries of $\Phi(s)$. Refer to (4.4). Also,

$$
\begin{aligned}
\zeta^{\star}(s) & =\zeta(s)\left(1-3^{-s}\right)^{-1} \cdot(2 \pi)^{1-s} \Gamma(s) \\
& =\sqrt{3} \cdot 3^{-s} \cdot \zeta^{\star}(1-s) .
\end{aligned}
$$

In (4.5), we used an important fact that $\theta_{j}(z)=\operatorname{Res}_{s=2 / 3} E_{j}(z, s)$ are in fact the same up to a scalar $a_{2 j}(7 / 6)$ at all eight cusps, similar to the case of S.J. Patterson [13], page 152 . $\tilde{L}(s)$.

In a manner similar to the case of $L(s)$, we can find the functional equation of

\section{The Main Theorem and an example}

It is a well known fact that the convolution of a cusp form with the quadratic theta series is analytic, i.e. the inner product of $f$ with the product of the quadratic theta series and its conjugate is zero. But in the cubic case we are presently investigating this inner product might or might not be zero. It is highly probable that it is non-zero. In fact one could probably prove this by a very unpleasant computation of the inner product. However, we choose the simpler approach of simply checking both alternatives.

We are now in a position to state the theorem. 
Theorem 5.1. Let $f$ be a weight 2 newform of $\Gamma_{0}(N)$ over $\mathbb{Q}, F$ be its lifting to $K=\mathbb{Q}(\sqrt{-3})$. Let

$$
L(s)=\sum_{m \in O_{K}} \frac{a_{m} \overline{\tau\left(\lambda^{-1} m\right)}}{(\mathcal{N} m)^{s}}, \tilde{L}(s)=N^{-2 / 3} \sum_{m \in O_{K}} \frac{a_{m} \tau\left(\lambda^{-1} N^{2} m\right)}{(\mathcal{N} m)^{s}},
$$

where $a_{m}$ and $\tau(m)$ are the Fourier coefficient of $F$ and the cubic theta series $\theta(z)$ respectively. If under the assumption $L(s)$ has no pole at $2 / 3, L(2 / 3) \neq \tilde{L}(2 / 3)$, then there are infinitely many cube free $M \in O_{K}$, such that

$$
L\left(1 / 2, F \otimes\left(\frac{\bullet}{M}\right)\right) \neq 0 .
$$

Proof. Let us recall that in Proposition 3.2, we proved $R_{0}(F, s, w)$ has a pole at $s=2 / 3$ with residue $L(w+1 / 6) \cdot G(w+1 / 6)$, and in Proposition 3.3, we computed that $R_{0}(F, s, w)$ has pole at $s=7 / 6-w$, with residue $(-1) \cdot \tilde{L}(7 / 6-w)$. $G(7 / 6-w) \cdot \frac{3}{4} \cdot(7 / 6-2 w)$. As an analytic function of two variables cannot vanish at an isolated point, we have

$$
\begin{aligned}
R_{0}(F, s, w)=\frac{L(w+1 / 6) G(w+1 / 6)}{s-2 / 3} & \\
& +\frac{(-1) \tilde{L}(7 / 6-w) \cdot G(7 / 6-w) \cdot(3 / 4) \cdot(7 / 3-2 w)}{s-(7 / 6-w)} \\
& + \text { analytic part, }
\end{aligned}
$$

where

$$
\begin{gathered}
L(s)=\sum_{m \in O_{K}} \frac{\tau\left(\lambda^{-1} m\right) a_{m}}{(\mathcal{N} m)^{s}}, \\
\tilde{L}(s)=N^{-2 / 3} \sum_{m \in O_{K}} \frac{\tau\left(\lambda^{-1} N^{2} m\right) a_{m}}{(\mathcal{N} m)^{s}},
\end{gathered}
$$

and $G(s)$ is as given in (3.15).

Define

$$
\alpha=\iint_{D} \operatorname{Res}_{s=7 / 6} J \cdot E_{\infty}^{3}(z, s) F(z) \cdot \operatorname{Res}_{s=2 / 3} \overline{E_{0}(z, \bar{s})} \frac{d x d y}{y^{3}}
$$

Changing variables $z \rightarrow \omega_{N} z$, and because the residues of the Eisenstein series satisfy the same transformation formulas as the Eisenstein series itself, we have

$$
\alpha=\iint_{D} \operatorname{Res}_{s=7 / 6} J \cdot E_{0}^{3}(z, s) F(z) \cdot \operatorname{Res}_{s=2 / 3} \overline{E_{\infty}(z, \bar{s})} \frac{d x d y}{y^{3}} .
$$

If $\alpha \neq 0$, then both $L(s)$ and $\tilde{L}(s)$ have simple pole at $s=2 / 3$, and by taking residues on both side of (3.13) at $w=1 / 2$, we have

$$
\underset{s=2 / 3}{\operatorname{Res}} L(s)=\frac{\alpha}{G(2 / 3)} .
$$

Thus

$$
L(s)=\frac{\alpha}{(s-2 / 3) G(2 / 3)}+\text { analytic part. }
$$


Similarly, we have

$$
\begin{gathered}
\operatorname{Res}_{s=2 / 3} \tilde{L}(s)=\frac{-\alpha}{G(2 / 3)}, \\
\tilde{L}(s)=\frac{-\alpha}{(s-2 / 3) G(2 / 3)}+\text { analytic part. }
\end{gathered}
$$

Now substituting (5.5) and (5.7) into (5.1), we have

$$
\begin{aligned}
R_{0}(F, s, w)= & \frac{\alpha}{(s-2 / 3)(s-7 / 6+w)}+\frac{\text { analytic part }}{s-2 / 3} \\
& +\frac{\text { analytic part }}{s-7 / 6+w}+\text { analytic part. }
\end{aligned}
$$

Thus, $R_{0}(F, s, w)$ has a double pole at $s=2 / 3, w=1 / 2$.

If $\alpha=0$, then $L(s)$ and $\tilde{L}(s)$ are both analytic at $s=2 / 3$ and (5.1) reads

$$
R_{0}(F, s, 1 / 2)=\frac{G(2 / 3)}{s-2 / 3}[L(2 / 3)-\tilde{L}(2 / 3)]+\text { analytic part. }
$$

If, under the assumption that $\tilde{L}(s)$ and $L(s)$ are both analytic at $s=2 / 3$, $\tilde{L}(2 / 3) \neq L(2 / 3)$, then $R_{0}(F, s, w)$ has a simple pole at $s=2 / 3, w=1 / 2$.

In both cases, $R_{0}(F, s, w)$ has poles at $s=2 / 3, w=1 / 2$. By (3.2), we know that $L(s, w)$ has a pole at the same point.

Now assuming Deligne's bound for the coefficients of holomorphic cusp forms, it is easily checked that the sum (over $d_{1}$ ) of all the terms with fixed $M$ in (3.11) converges absolutely at $s=2 / 3, w=1 / 2$ and that the sum (over $M$ ) of all the terms with the same cubic free part in (3.11) converges absolutely at the same point. Thus we conclude that there are infinitely many cubic free $M$, such that $L(1 / 2, F \otimes(\dot{\bullet})) \neq 0$. The proof is thus completed.

Now let us describe a method to compute $L(2 / 3)$ under the assumption that $L(s)$ has no pole at $2 / 3$.

We prove the following bound for $L_{j}(s)$, assuming Deligne's bound for the coefficients of holomorphic cusp forms. Let $\operatorname{Re} s>1$, and let $\sigma_{0}(m)=$ number of factors of $m$. Then

$$
\begin{aligned}
\left|L_{j}(s)\right| & =\left|\sum_{m \in O_{K}} \frac{a_{m}^{j} \tau\left(\lambda^{-1} m\right)}{(\mathcal{N} m)^{s}}\right| \\
& \leq \sum_{m \in O_{K}} \frac{\sigma_{0}(m)\left|\tau\left(\lambda^{-1} m\right)\right|}{(\mathcal{N} m)^{\text {Res }}} .
\end{aligned}
$$

Here the functions $\sigma_{0}$ and $|\tau|$ are multiplicative. After computing the $p$-factors we can prove the following proposition.

\section{Proposition 5.2.}

$$
\left|L_{j}(s)\right| \leq 2 \cdot \sqrt{3} \cdot 27 \cdot \zeta(3 s-1 / 2) \frac{\zeta(s)}{\zeta(2 s)} \frac{1+2 \cdot 3^{1 / 2-3 s}+2 \cdot 3^{-1 / 2-s}+3^{-4 s}}{\left(1+3^{-s}\right)^{2}},
$$

where $L_{j}(s)$ is as given in (4.6) and $a_{m}^{j}$ are the coefficients of $F$ at the cusp $j$.

We will make use of the following lemma from T.M. Apostol [1], page 281, Lemma 3 . 
Lemma 5.3.

$$
\frac{1}{2 \pi i} \int_{\substack{\alpha-i \infty \\ \alpha>0}}^{\alpha+i \infty} \frac{H^{s} d s}{s(s+1 / 2) \cdots(s+r / 2)}= \begin{cases}\frac{2^{r}}{r !}\left(1-\frac{1}{\sqrt{H}}\right)^{r}, & H>1, \\ 0, & 0 \leq H \leq 1 .\end{cases}
$$

Let us write

$$
\begin{aligned}
& l(s)=\sum_{n=1}^{\infty} \frac{b_{n}}{n^{s}}=L(s) \cdot \zeta(6 s-2) \cdot\left(1-3^{2-6 s}\right)^{-1}, \\
& g(s)=\left(3^{10} \cdot 2^{-8} \cdot \pi^{-8}\right)^{s} \cdot \Gamma(2 s-2 / 3) \cdot \Gamma(2 s-1 / 3) \cdot \Gamma^{2}(s+1 / 3) \cdot \Gamma^{2}(s+2 / 3) .
\end{aligned}
$$

The functional equation (4.5) can be rearranged as follows:

$$
l(s) g(s)=\sum_{j=1}^{8} a_{1 j}(s+1 / 2) a_{2 j}(7 / 6) l_{j}(1-s) g(1-s) \cdot \frac{1-s}{s} \cdot 3^{3-6 s},
$$

where $l_{j}(s)=L_{j}(s) \cdot \zeta(6 s-2) \cdot\left(1-3^{2-6 s}\right)^{-1}, a_{i j}(s)$ are entries of $\Phi(s)$, which is the scattering matrix.

Then by the lemma we have

$$
\begin{aligned}
I N T & =\frac{1}{2 \pi i} \int_{1-i \infty}^{1+i \infty} \frac{l(s+2 / 3) x^{s} d s}{s(s+1 / 2) \cdots(s+r / 2)} \\
& =\frac{2^{r}}{r !} \sum_{n \leq x} \frac{b_{n}}{n^{2 / 3}}\left(1-\sqrt{\frac{n}{x}}\right)^{r},
\end{aligned}
$$

where as $\operatorname{Re}(s+2 / 3)=5 / 3>1$ implies that the series for $l(s+2 / 3)$ converges absolutely.

On the other hand moving the line of integration to the left

$$
\begin{aligned}
I N T=\frac{1}{2 \pi i} & \int_{\substack{-\beta-i \infty \\
\beta=2 / 3+\delta_{0} \\
1 / 3>\delta_{0}>0}}^{-\beta+i \infty} \frac{l(s+2 / 3) x^{s} d s}{s(s+1 / 2) \cdots(s+r / 2)}+\frac{l(2 / 3) 2^{r}}{r !} \\
& +\frac{l(1 / 6) x^{-1 / 2}}{(-1 / 2)(1 / 2) \cdots(\{r-1\} / 2)} .
\end{aligned}
$$

Set $s=1 / 6$ in (5.11). Then the right hand side of the equation is analytic, because both $l_{j}(5 / 6)$ and $g(5 / 6)$ are analytic. In the left hand side of the equation $g(s)$ has a pole at $1 / 6$. Thus we conclude that $l(1 / 6)=0$.

Set $r=10, \delta_{0}=1 / 16$.

Let

$$
B=\frac{1}{2 \pi i} \int_{\substack{-\beta-i \infty \\ \beta=35 / 48}}^{-\beta+i \infty} \frac{l(s+2 / 3) x^{s} d s}{s(s+1 / 2) \cdots(s+r / 2)} .
$$


Then

$$
\begin{aligned}
|B| & =\left|\frac{1}{2 \pi i} \int_{\substack{\beta-i \infty \\
\beta=35 / 48}}^{-\beta+i \infty} \frac{l(s+2 / 3) x^{s} d s}{s(s+1 / 2) \cdots(s+r / 2)}\right| \\
& =\left|\frac{1}{2 \pi i} \int_{t=-i \infty}^{\infty} \frac{l\left(-\delta_{0}+i t\right) x^{-35 / 48+i t} d t}{\left(\frac{-35}{48}+i t\right)\left(\frac{-35}{48}+i t+\frac{1}{2}\right) \cdots\left(\frac{-35}{48}+i t+\frac{r}{2}\right)}\right| \\
& \leq \frac{M}{\pi} x^{\frac{-35}{48}} \int_{0}^{\infty} \frac{\left|\frac{g\left(1+\delta_{0}+i t\right)}{g\left(-\delta_{0}+i t\right)}\right| d t}{\left|\prod_{i=1}^{11}\left(\frac{-35}{48}+i t+\frac{i-1}{2}\right)\right|} \\
& =\alpha(N) \cdot x^{\frac{-35}{48}} .
\end{aligned}
$$

Note that by (5.11) and the Proposition 5.2, we have

$$
l\left(-\delta_{0}+i t\right) \leq M\left|\frac{g\left(1+\delta_{0}+i t\right)}{g\left(-\delta_{0}+i t\right)}\right|
$$

where

$$
\begin{gathered}
M=\operatorname{Max}_{t} \tilde{\Phi}(7 / 16+i t) \cdot \tilde{l}(1+1 / 16) \cdot\left|\frac{17 / 16+i t}{1 / 16+i t}\right| \cdot\left|3^{3-6(-1 / 16+i t)}\right|, \\
\tilde{l}(s)=2 \cdot \sqrt{3} \cdot 27 \cdot \zeta(3 s-1 / 2) \cdot \zeta(6 s-2) \cdot \frac{\zeta(s)}{\zeta(2 s)} \\
\cdot \frac{1+2 \cdot 3^{1 / 2-3 s}+2 \cdot 3^{-1 / 2-s}+3^{-4 s}}{\left(1+3^{-s}\right)^{2}\left(1-3^{2-6 s}\right)}, \\
\tilde{\Phi}(s)=\left(\sum_{j=1}^{8}\left|a_{1 j}(s+1 / 2) a_{2 j}(7 / 6)\right|\right)\left|\frac{3^{6 s-6}-1}{3^{6 s-5}-1}\right|
\end{gathered}
$$

The constant $\alpha(N)$ in the last line of (5.14) can be computed by a simple Mathematica program. For instance $\alpha(11)=0.18$.

Combining (5.12) and (5.13), we have

$$
l(2 / 3)=\sum_{n=1}^{x} \frac{b_{n}}{n^{2 / 3}}\left(1-\sqrt{\frac{n}{x}}\right)^{r}-\frac{10 !}{2^{10}} B .
$$

Now observe that when $x \rightarrow \infty,|B| \rightarrow 0$; thus (5.15) will always give us the ever wanted accuracy.

The same method can be used to compute $\tilde{L}(2 / 3)$ under the assumption that $\tilde{L}(s)$ is analytic at $2 / 3$.

Example. Let $f=\eta^{2}(\tau) \eta^{2}(11 \tau)$ be the newform of weight 2 level 11 . Using Mathematica, set $x=1000$; we have $l(2 / 3)=\zeta^{\star}(2) L(2 / 3)=105.92+C$, with $|C|<$ 4.14. $\tilde{l}(2 / 3)=\zeta^{\star}(2) \tilde{L}(2 / 3)=9.57+C$, with $|C|<4.14$.

Thus the hypothesis of the theorem is satisfied and so there are infinite many $M \in O_{K}$, such that $L(1 / 2, F \otimes(\dot{\dot{M}})) \neq 0$, where $F$ is the lifting of $f$ over $\mathbb{Q}(\sqrt{-3})$. 


\section{ACKNOWLEDGEMENTS}

The results of this paper are from the author's Brown University Ph.D. thesis. I would like to thank my advisor Jeffrey Hoffstein for his great help. I would also like to thank the referee for the valuable comments.

\section{REFERENCES}

1. Tom M. Apostol, Introduction to Analytic Number Theory, Undergraduate Texts in Mathematics, Springer-Verlag (1976). MR 55:7892

2. D. Bump, S. Friedberg and J. Hoffstein, Eisenstein series on the metaplectic group and nonvanishing theorems for automorphic L-functions and their derivatives, Annals of Math. 131 53-127 (1990). MR 92e: 11053

3. D. Bump, S. Friedberg and J. Hoffstein, Nonvanishing theorems for L-functions of modular forms and their derivatives, Inventiones Math. 102 543-618 (1990). MR 92a:11058

4. D. Bump and J. Hoffstein, Cubic metaplectic forms on GL(3), Inventiones Math. 84 481-505 (1986). MR 87i:11059

5. S. Friedberg and J. Hoffstein, Nonvanishing theorems for automorphic L-functions on GL(2), Annals of Math. 142 385-423 (1995). MR 96e:11072

6. S. Friedberg, On the imaginary quadratic Doi-Naganuma lifting of modular forms of arbitrary level, Nagoya Math. J. 92 1-20 (1983). MR 85f:10031

7. D. Goldfeld, J. Hoffstein and S. Patterson, On automorphic functions of half-integral weight with applications to elliptic curves, in Number Theory related to Fermat's Last Theorem (N. Koblitz, ed.), Birkhauser, Boston, Basel, Stuttgart, 153-193 (1983). MR 84i:10031

8. I. Gradshteyn and I. Ryzhik, Tables of integral series and products, Fifth edition. MR 94g:00008

9. K. Ireland and M. Rosen, A classical introduction to modern number theory, Graduate Texts in Mathematics, Springer-Verlag 84 (1982). MR 83g:12001

10. H. Iwaniec, On the order of vanishing of modular L-functions at the critical point, Seminaire de Theorie des Nombres, Bordeaux 2 365-376 (1990). MR 92h:11040

11. D. Lieman, Nonvanishing of L-series associated to cubic twists of elliptic curves, Annals of Math. 140 81-108 (1994). MR 95g:11044

12. K. Murty and R. Murty, Mean values of derivatives of modular L-series, Annals of Math. 133 447-475 (1991).

13. S.J. Patterson, A cubic analogue of the theta series, J. Reine Agnew Math. 296 125-161 (1977). MR 58:27795b

14. D. Rohrlich, Nonvanishing of L-functions for GL(2), Inventiones Math. 97 381-403 (1989). MR 90g:11062

15. G. Shimura, On the periods of modular forms, Math. Annalen 229 211-221 (1977). MR 57:5911

16. J. Waldspurger, Correspondences de Shimura et quaternions, Forum Math 3 219-307 (1991). MR 92g: 11054

Department of Mathematics, The Pennsylvania State University, University Park, PenNSYLVANia 16802

Current address: Financial Data Planning Corp., 2140 S. Dixie Hwy., Miami, Florida 33133

E-mail address: xiaoties@fdpcorp.com 